\title{
Über die Löslichkeit von Salzgemisehen in Wasser.
}

\section{Von}

\section{Guido Bodländer.}

Bei der Untersuchung der Löslichkeit von Rohrzucker und einigen Salzen in Gemischen von Allohol und Wasser ${ }^{1}$ ) hatte sich herausgestellt, dass das Wasser um so weniger von den in Alkohol unlöslichen, in Wasser löslichen Körpern aufzunehmen im stande ist, mit je mehr Alkohol es vermischt ist. Die Löslichkeitsverminderung konnte einem spezifischen Einfluss des Alkohols nicht zugeschrieben werden, und es war die Annahme gemacht worden, dass der Alkohol nur als Verdïnnungsmittel wirke, und dass das Wasser um so weniger von einem darin löslichen Körper aufnehme, je stärlker es mit einem für den letzteren indifferenten Stoff rerdünnt sei. Ist diese Amnahme richtig, dann darf nicht nur ein Zusatz von Alkohol das Lösungsvermögen des Wassers für ein Salz herabsetzen, auch ein Zusatz eines beliebigen anderen Körpers, der auf das Salz weder chemisch wirkt, noch mit ihm Doppelsalze oder isomorphe Mischungen bildet, muss den nämlichen Einfluss haben. Dass das Lösungsvermögen des Wassers für Salze herabgedrückt wird, wenn in demselben andere gegen die ersten indifferente Salze bis zur Sättigung aufgelöst sind, ist durch zahlreiche Untersuchungen von Karsten $\left.\left.\left.{ }^{2}\right), K_{o p p}{ }^{3}\right), H_{a n s e n}^{4}\right)$, Riidorff ${ }^{5}$ u. a. festgestellt worden. Bernht diese Herabsetzung auf denselben Grïnden wie die Herabsetzung des Lösungsvermögens des Wassers durch Alkohol, also auf einer Verdünnung des Lösungsmittels, so muss sie auch denselben Gesetzen folgen und die für jene Gemische aufgestellte Regel, dass in den verschiedenen bei gleicher Temperatur gesättigten Lösungen eines Stoffes der Quotient aus der Menge des in einem bestimmten Volumen der Lösung

1) Vgl. diese Zeitschr. 7, 208 (1891). 1840, S. 95 . 3) Lieb. Ann. 34, 260 (1840). 98, 137 (1866); 103, 114 (1868).
2) Sitzungsber, d. Berl. Akad.

4) Journ. f, prakt. Chemie 5) Pogg. Ann. 148, 456, 555 (1873). 
enthaltenen Wassers durch die Kubikwurzel aus der Menge des gelösten Stoffes eine konstante Grösse ist, muss auch für die gesättigten Lösungen eines Salzes in Wasser, das wechselnde Menge eines anderen für das erste indifferenten Salzes enthält, Geltung haben.

Zur Prifung dieses Satzes konnte das reiche Beobachtungsmaterial über die Löslichkeit von Salzgemischen, welches von den oben genannten Autoren geliefert worden ist, nicht benutzt werden, weil bis auf wenige Ausnahmen immer nur die Löslichkeitsverhältnisse in solchen Lösungen festgestellt worden sind, die für beide Salze gesättigt waren, und weil ferner die Angaben über die spezifischen Gewichte der Lösungen, bezw. über die in bestimmten Volumen enthaltenen Mengen der drei Körper fehlen. Es wurden deshalb vier Versuchsreihen angestellt, in denen ich gesättigte wässerige Lösungen von

1. Chlorkalium mit wechselnden Mengen Kaliumnitrat,

2. Kaliumnitrat "

3. Chlornatrium "

$\eta$

4. Natriumnitrat"

$"$

$\eta$

Chlorkalium,

Natriumnitrat,

Chlornatrium

versetzte und die Zusammensetzung der entstehenden Löstungen untersuchte.

In jeder Versuchsreihe wurde von dem Salze, dessen Löslichkeit untersucht werden sollte, eine grössere Menge der gesättigten wässerigen Lösung hergestellt, und von dieser wurden gleiche Mengen mit steigenden Mengen des anderen fein pulverisierten Salzes versetzt, und dessen Lösung wurde durch Umschütteln und Erwärmung bewirkt. Dabei schied sich nach dem Erkalten immer etwas von dem ersten Salze aus; um die Lösungen an diesem gesättigt zu erbalten, wurden sie mit dem fein gepulvertem Salze versetzt und bei konstanter T'emperatur unter häufigem Umschütteln, mindestens 24 Stunden lang, auf bewahrt. Dann wurden alle Lösungen einer Versuchsreihe nach vorangegangenem, langem Schütteln durch trockene Filter in trockene Gefüsse gleichzeitig filtriert, das spezifische Gewicht der Lösungen wurde mit der Westphalschen Wage bei der Sättigungstemperatur ermittelt, und ein Teil der Flüssigkeit wurde für die Analyse abgewogen und abgemessen. Die Analyse erfolgte durch vorsichtiges Eindampfen, gelindes Glühen und Wägung des Salzgemisches, Auflösung desselben in Wasser und Titrieren des Chlors nach Volhard. Die aus dem. Chlorgehalt berechnete Menge des Chlorids liess aus der Differenz gegen den Gesamtritckstand das Nitrat ermitteln; das Wasser ergab sich aus der Differenz der Einwage gegen den Ritckstand. 
I. Löslichkeit von Chlorkalium auf Zusatz von Kaliumnitrat.

Sättigungstemperatur $175^{\circ}$.

\begin{tabular}{|c|c|c|c|c|c|c|}
\hline $\mathrm{Nr}$. & $\begin{array}{l}\text { spez. Gew. } \\
\text { der Lousung }\end{array}$ & $\begin{array}{c}100 \mathrm{ccm} \\
K O l \\
S\end{array}$ & $\begin{array}{c}\text { der Lösung } \\
\text { Wasser } \\
W\end{array}$ & $\begin{array}{l}\text { enthalten } \\
\qquad \mathrm{KNO}_{3}\end{array}$ & $\frac{100 K C l}{W}$ & $\frac{W}{\sqrt[3]{S}}$ \\
\hline 1 & $1 \cdot 1730$ & $29 \cdot 39$ & $87 \cdot 85$ & 0 & $33 \cdot 45$ & $28 \cdot 47$ \\
\hline 2 & $1 \cdot 1980$ & $27 \cdot 50$ & $85 \cdot 68$ & $6 \cdot 58$ & $32 \cdot 09$ & $28 \cdot 39$ \\
\hline 3 & $1 \cdot 2100$ & $27 \cdot 34$ & $84 \cdot 76$ & $8 \cdot 83$ & 32.23 & $28 \cdot 14$ \\
\hline 4 & 1.2250 & 26.53 & $83 \cdot 58$ & $12 \cdot 48$ & $31 \cdot 73$ & $28 \cdot 02$ \\
\hline 5 & $1 \cdot 2360$ & $25 \cdot 98$ & $82 \cdot 84$ & 14.83 & $31 \cdot 32$. & $27 \cdot 97$ \\
\hline 6 & $1 \cdot 2390$ & $25 \cdot 96$ & $82 \cdot 65$ & $15 \cdot 22$ & $31 \cdot 39$ & $27 \cdot 91$ \\
\hline 7 & $1 \cdot 2388$ & 25.95 & $82 \cdot 43$ & $15 \cdot 49$ & $31 \cdot 50$ & $27 \cdot 84$ \\
\hline 8 & $1 \cdot 2410$ & $26 \cdot 24$ & $82 \cdot 68$ & $15 \cdot 33$ & $31 \cdot 75$ & $27 \cdot 81$ \\
\hline
\end{tabular}

In den vier letzten Lösungen hatten sich Krystalle von Kalisalpeter ausgeschieden; die Lösungen waren also auch mit diesem Salze ganz oder nahezu gesättigt.

II. Löslichkeit von Kaliumnitrat auf Zusatz von Chlorkalium.

Sättigungstemperatur $205^{\circ}$.

$100 \mathrm{ccm}$ der Lösung enthalten $100 \mathrm{~S}$

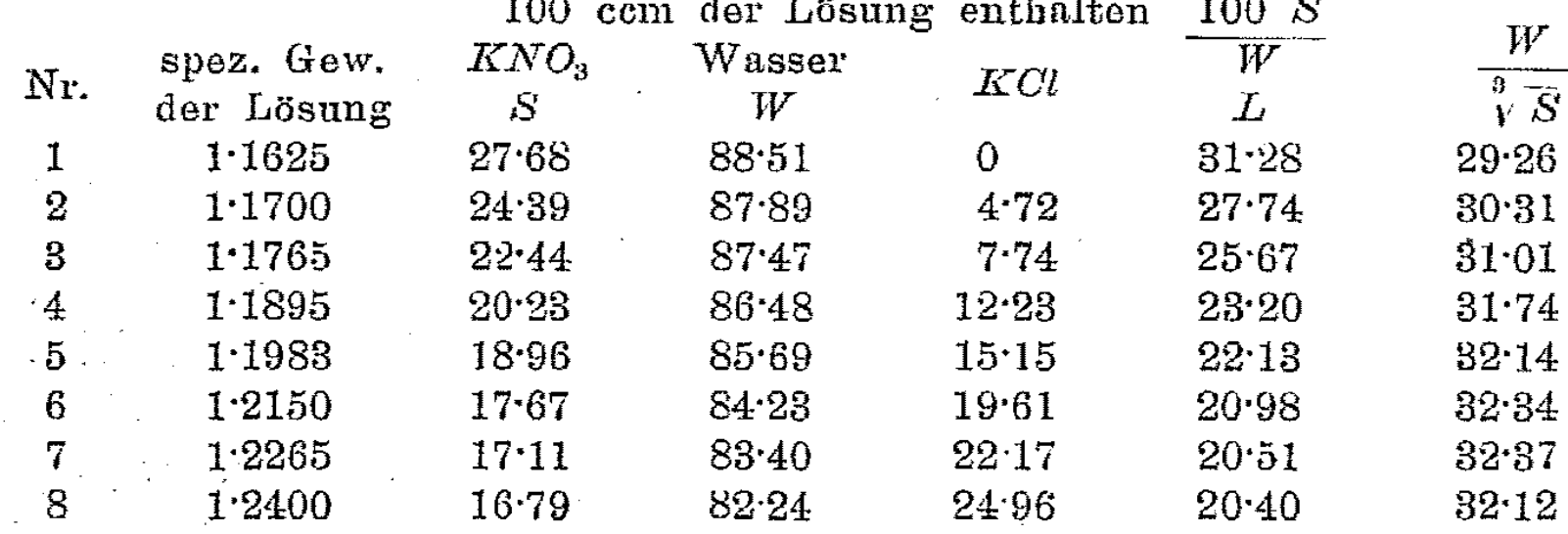

III. Iöslichkeit von Chlornatrium anf Zusatz von Natriumnitrat.

Sattigungstemperatur $15 \cdot 5^{\circ}$.

$100 \mathrm{ccm}$ Lösung enth. $100 \mathrm{~S}$

Nr. spez. Gew. NaCl Wasser $\mathrm{NaNO}^{-}$

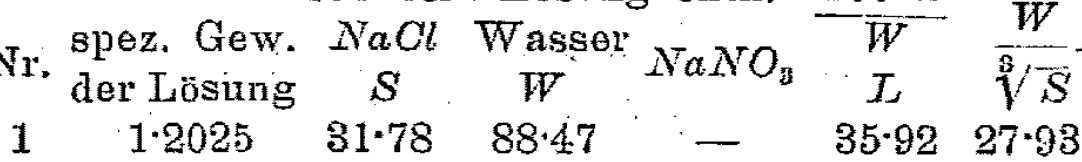

2. $1.2305 \quad 27 \cdot 89$

3. $1 \cdot 2580 \cdot 26 \cdot 31$

$4 \quad 1 \cdot 2810 \quad 23 \cdot 98$

$5 \quad 1.3090 \quad 22 \times 30$.

$6 \quad 1 \cdot 3345 \quad 20 * 40$

$7 \cdot 1 \cdot 3465$

$8 \quad 1 \cdot 3465$
$87 \cdot 63$

$86 \cdot 25$

$82 \cdot 66$

$80 \cdot 42$

79.25

$19 \cdot 40$

$19 \cdot 67$
$77 \cdot 37$

$77 \cdot 34$
$35.92 \quad 27.93$

$7 \cdot 53, \quad 31 \cdot 92 \quad 28 \cdot 90$

$13 \cdot 24 \quad 30 \cdot 50 \cdot 29 \cdot 00$

$21.58 \quad 29.00 \quad 28.66$

$\begin{array}{lll}28 \cdot 18 & 27 \cdot 74 & 28 \cdot 57\end{array}$

$33 \cdot 80$

37.88

$25 \cdot 74 \quad 29 \cdot 00$

$25 \cdot 08 \quad 28 \cdot 79$

$\begin{array}{lll}37.64 & 25.40 & 28.65\end{array}$
$100 \mathrm{ccm}$ Lösung enth. $100 \mathrm{~S}^{\circ}$

$\mathrm{NaCl}+2 \mathrm{H}_{2} \mathrm{O}$ Wasser $\frac{\mathrm{W}^{r}}{}$

$S^{\prime}$

$51 \cdot 34$

$W^{\prime}$

$L^{\prime}$

$\frac{W^{\prime}}{\sqrt[8]{S^{\prime}}}$

68.91

74.50

$18 \cdot 54$
$45 \cdot 05$

$42 \cdot 50$

$33 \cdot 74$

$70 \cdot 47$

70.06

$67 \cdot 90$

63.91

$60 \cdot 66$

$57 \cdot 04$

$54 \cdot 00$

$49 \cdot 40$

47.90

$48 \cdot 70$

$19 \cdot 81$

20.08

20.07

$32 \cdot 95$

$31 \cdot 34$

$31 \cdot 77$
$66 \cdot 70$

$65 \cdot 43$

$65 \cdot 24$
$20 \cdot 20$

$20 \cdot 81$

$20 \cdot 76$

$20 \cdot 60$ 
Aus den beiden letzten Lösungen hatten sich Rhomboëder von Natriumnitrat ausgeschieden; diese Lösungen waren also mit beiden Salzen gesättigt.

IV. Löslichkeit von Natriumnitrat auf Zusatz von Chlornatrium.

Suttigungstemperatur $15^{\circ}$.

\begin{tabular}{|c|c|c|c|c|c|c|}
\hline \multirow[b]{2}{*}{$\mathrm{N}$. } & \multirow[b]{2}{*}{$\begin{array}{l}\text { spez. Gew. } \\
\text { der Lösung }\end{array}$} & \multicolumn{3}{|c|}{$100 \mathrm{ccm}$ der Lösung enthalten } & $100 \mathrm{~S}$ & \multirow[b]{2}{*}{$\frac{W}{\sqrt[3]{S}}$} \\
\hline & & $\begin{array}{c}\mathrm{NaNO}_{3} \\
\mathrm{~S}\end{array}$ & $\begin{array}{c}\text { Wasser } \\
W\end{array}$ & $\mathrm{NaCl}$ & $\begin{array}{l}W \\
L\end{array}$ & \\
\hline 1 & $1 \cdot 3720$ & $62 \cdot 38$ & $74: 82$ & 0 & $82 \cdot 38$ & 1887 \\
\hline 2 & $1 \cdot 3645$ & $56 \cdot 76$ & $75 \cdot 69$ & $4 \cdot 00$ & $74 \cdot 86$ & $19 \cdot 70$ \\
\hline 3 & $1 \cdot 3585$ & $59 \cdot 09$ & $75 \cdot 71$ & $7 \cdot 24$ & $68 \cdot 60$ & $20 \cdot 27$ \\
\hline 4 & 1.3530 & $47 \cdot 08$ & $76 \cdot 86$ & $11 \cdot 36$ & $61 \cdot 25$ & $21 \cdot 29$ \\
\hline 5 & 1:3495 & $42 \cdot 66$ & 76.96 & $15 \cdot 33$ & 55.43 & $21 \cdot 99$ \\
\hline 6 & $1 \cdot 3485$ & $39 \cdot 90$ & $77 \cdot 14$ & $17 \cdot 81$ & $51 \cdot 72$ & $22 \cdot 58$ \\
\hline 7 & $1 \cdot 3485$ & $38 \cdot 73$ & $77 \cdot 15$ & 18.97 & $50 \cdot 20$ & $22 \cdot 80$ \\
\hline 8 & $1+3485$ & $38 \cdot 02$ & $77 \cdot 49$ & $19 \cdot 34$ & 49.06 & 23.05 \\
\hline
\end{tabular}
schieden.

In den zwei letzten Versuchen hatten sich Kochsalzwürfel ausge-

Aus den vier Versuchsreihen ergiebt sich, dass das Lösungsvermögen des Wassers für Chlorkalium, Kaliumnitrat, Chlornatrium und Natriumnitrat abnimmt, wenn in dem Wasser ein anderes Salz auch nur in kleinen Mengen gelöst ist. Die Löslichkeitsabnahme ist um so grösser, je mehr von dem fremden Salze in der Lösung vorhanden ist.

Aber nicht nur hierin zeigt sich die Analogie in der Wirkung des Salzzusatzes mit der Wirkung eines Alkoholzusatzes, sie tritt in den ersten drei Versuchsreihen auch quantitativ darin hervor, dass für jedes Salz in den verschiedenen mit demselben gesättigten Lösungen das Verhältnis $\frac{W}{\sqrt[3]{S}}$ angenähert konstant bleibt. Sowohl der Alkohol wie ein fremdes indifferentes Salz wirken demnach wesentlich nur verdünnend auf das Lösungsmittel; eine spezifische Wirkung kommt weder dem einen, noch dem anderen in starkem Grade zu, die Hauptursache der durch sie ausgeübten Löslichkeitsverminderung ist die, dass sie durch ihre Raumausfülling die Menge des in einem bestimmten Volumen enthaltenen Lösungsmittels und damit anch die Menge des gelösten Stoffes vermindern, des letzteren aber nicht im proportionalen Verhältnis, sondern so, dass der Wert Wasser: $\sqrt[3]{\mathrm{Salz}}$ einen konstanten Wert behält.

Eine Ausnahme von dieser Regel bildet die vierte Versuchsreihe; hier steigen die Zahlen der letzten Spalte stetig, von 18.87 bis 23.05 , während bei den Versuchen, in denen die Löslichkeit des Natriumnitrats 
durch Alkohol- statt durch Kochsalzzusatz herabgedrückt war, die entsprechenden Zahlen nur sehr wenig sich ïber den Ausgangswert erheben. Eine Erklärung dieser Abweichung bietet sich, wenn man die Wassermengen berechnet, die, wenn die Konstanz des Wertes $\frac{W}{\sqrt[3]{S}}$ auch hier Geltung hätte, mit dem Natriumnitrat hätten verbunden sein müssen. Es ergeben sich folgende Zahlen.

$\begin{array}{lllll}\text { Lösung } & 1 & 2 & .3\end{array}$

Wasser berechnet

Wasser gefunden

34

$74 \cdot 82 \quad 75 \cdot 69$

$70 \cdot 46$

$68 \cdot 12$

5

$75 \cdot 71$

$76 \cdot 86$

6592

6

7

8

Differenz

$0 \quad 3 \cdot 19$

$5 \cdot 25$

$8 \cdot 74$

$76 \cdot 96$

$64 \cdot 47$

$63 \cdot 83$

$63 \cdot 44$

Chlornatrinm in

der Lüstung

$0 \quad 4.00$

$7 \cdot 24$

$11 \cdot 36$

11.04

$77 \cdot 14$

$77 \cdot 15$

$77 \cdot 49$

$12 \cdot 67 \quad 13 \cdot 32 \quad 14.05$

Differenz

Chlornatrium

0.80

0.72

0.77

$15 \cdot 33$

$17 \cdot 81$

$18 \cdot 97$

$19 \cdot 34$

0.72

0.71

$0 \cdot 70$

0.72

Die Differenz des berechneten gegen das gefundene Wasser ist also nahe der Menge des Chlornatriums proportional; dies Verbalten findet eine Frklärrung, wenn man annimmt, dass das Chlornatrium einen Teil des Wassers fest als Hydratwasser bindet, und dass das Hydratwasser nicht als Lösungsmittel für das Natriumnitrat dient. Belkanntlich existiert ein unter $0^{0}$ beständiges, monoklin krystallisierendes Hydrat $\mathrm{NaCl}+2 \mathrm{H}_{2} \mathrm{O}$ in festem Zustande, die Anwesenheit desselben Hydrats in Lösungen haben Ridorff $f^{1}$ und Coppet ${ }^{2}$ ) aus ihren Untersuchungen ïber die Gefrierpunktserniedrigung gefolgert. Die Beobachtungen iiber die Löslichkeit von Kochsalz in Gemischen ron Alkohol und Wasser schliessen sich ebenfalls weit besser an die bei den anderen Salzen erhaltenen Resultate unter der Annahme an, dass das Kochsalz hier als Hydrat mit zwei Molekiilen Wasser in den Lösungen vorhanden sei. In einem solchen Hydrat wären mit einem Gramm $\mathrm{NaCl} 0.6154 \mathrm{~g} \mathrm{H}_{2} \mathrm{O}$ verbunden; die oben durchgeführte Berechnung des in den Natriumnitrat-Chlornatriumlösungen als an Chlornatrium gebunden angenommenen Wassers ergiebt für $1 \mathrm{~g} \mathrm{NaCl} 0.7-0.8 \mathrm{~g} \mathrm{H}_{2} \mathrm{O}$, also eine etwas grössere Zahl. Das dảrf aber nicht befremden, denn bei der Berechnung war von der absoluten Konstanz des Wertes $\frac{W}{\sqrt[3]{S}}$ ausgegangen; bei den anderen Salzgemischen und bei den Lösungen von Natriumnitrat in Alkoholwassergemischen steigen aber diese Werte zunächst mit zu-

1) Pogg. Ann. 114, 63 (1861), 116, 55 (1862) und 145, 599 (1871).

2) Ann. Chim. Phys. [4] 23, 866 (1871), 25, 502 (1872) u. 26, 98 (1872). 
nehmender Menge des fremden Körpers. Eine solche Steigerung ergiebt sich auch, wenn man fïr die Natriumnitrat-Chlornatriumlösungen die Werte $\frac{W^{\prime}}{\sqrt[3]{S}}$ berechnet unter der Annahme, dass das Chlornatrium zwei Molekïle Wasser fest bindet, so dass nur der Rest - $W^{\prime}-$ als Lösungsmittel für Natriumnitrat dient.

$\begin{array}{lcccccccc}\text { Lösung } & 1 & 2 & 3 & 4 & 5 & 6 & 7 & 8 \\ W^{\prime} & 74.82 & 73.28 & 71.26 & 69.87 & 67.53 & 66 \cdot 18 & 65.48 & 65.59 \\ \frac{W^{\prime}}{\sqrt[3]{S}} & 18.87 & 19.06 & 19.08 & 19.35 & 19.33 & 19.37 & 19.35 & 19.51\end{array}$

Die Zahlenreihe $\frac{W^{\prime}}{\sqrt[3]{S}}$ verläuft sehr nahe parallel derjenigen der entsprechenden Werte in der zweiten Versuchsreihe, betreffend die Löslichkeit von Natriumnitrat in Gemischen von Alkohol und Wasser ${ }^{1}$ ), und damit ist die scheinbare Ausnahme beseitigt, welche das Verhalten der Natriumnitrat-Chlornatriummischungen darbot. Durch den Zusatz der vier Salze in den vier Versuchsreihen ist die Löslichleit der Salze, mit denen die Lösungen gesättigt waren, in fast derselben Weise herabgedrückt worden wie durch Zusatz von Alkohol.

Wenn die Löslichkeitsverminderung eines Salzes, z. B. des Chlornatriums, durch den Alkohol- und durch den Natriumnitratzusatz nur vermöge der Verdünnmng des Lösungsmittels bewirkt wïrde, so müsste in den bei gleicher 'Temperatur gesättigten Lösungen beider Versuchsreihen gleichem Wassergehalt anch ein genan gleicher Gehalt an Chlornatrium entsprechen, weil sonst die Werte $\frac{W}{\sqrt[3]{S}}$ nicht in beiden Fällen den gleichen Verlauf zeigen könnten. Nun sind zwar die beiden Versuchsreihen, die am besten mit einander verglichen werden können, die zweite über die Löslichkeit von Chlomatrium auf Zusatz von Alkohol ${ }^{2}$ ) und die über die Löslichkeit auf Zusatz von Natriumnitrat nicht bei gleicher Temperatur angestellt worden $(11.5$ und $15.5 \%$, aber die Temperatur besitzt keinen grossen Einfluss auf die Löslichkeit von Chlornatrium, so dass ein direlkter Vergleich doch zulässig erscheint. Berechnet man durch Interpolation die in beiden Versuchsreihen gleichen Wassermengen entsprechenden Kochsalzmengen, so ergeben sich folgende Zahlen:

1) Diese Zeitschr. 7, 318 (1891).

2) Diese Zeitsch: 7, 317 (1891). 
Gehalt

Wasser in $100 \mathrm{ccm}$ beider Lösungen

88.5

$87 \cdot 6$

87

86

85

84

83

82

81

80

79

78
Gehalt an Kochsalz in der Lösung mit Natriumnitrat

mit Alkohol

$31 \cdot 6$

$31 \cdot 0$

$30 \cdot 5$

$29 \cdot 6$

28.8

$27 \cdot 9$

$27 \cdot 2$

26.4

$25 \cdot 8$

$25 \cdot 1$

$24 \cdot 1$

$23 \cdot 3$
Differenz

0

$3 \cdot 1$

$3 \cdot 3$

3.4

$3 \cdot 4$

$3 \cdot 1$

$3 \cdot 0$

2.9

$3 \cdot 1$

$3 \cdot 2$

35

$3 \cdot 3$

Gleiche Mengen Wasser lösen also bei gleicher, durch Natriumnitrat hervorgerufener Verdünnung weniger Chlornatrium auf, als wenn die Verdïnnung durch Alkohol bewirkt wird. Schon der erste Zusatz des Natriumnitrats bringt die Differenz zwischen dem Lösungsvermögen beider Flüssigkeiten hervor, und diese Differenz bleibt bei ferneren Zusätzen in sehr nahe gleicher Höhe bestehen.

Eine Erklärung für dieses Verhalten bietet sich in den Versuchen und Anschauungen von Nernst ${ }^{1}$ ) über die Löslichkeit von Salzgemischen. Nernst hat nur den Faktor der Löslichkeitsverminderung berücksichtigt, der von der Dissociation der beiden Salze mit gemeinsamem Ion herrïbrt. In den von ilum und von Noyes ${ }^{2}$ ) angestellten Versuchen mit wenig löslichen. Salzen von weit vorgeschrittener Dissociation sind Löslichkeitsverminderungen beobachtet worden, die durch die Verdïnnung des Lösungsmittels allein nicht erklärt werden können und nahezu übereinstimmen mit den nach den Dissociationsgesetzen berechneten Löslichkeitsverminderungen. Bei der Löslichkeit von Gemischen leicht löslicher Körper kommt aber die Wirkung des nicht dissociierten und des dissociierten Anteils als Verdünnungsmittel für das Wasser zu der ron Nernst geforderten Proportionalität zwischen der aktiven Masse des undissociierten Stoffes und dem Produkt der aktiven Massen seiner Dissociationsprodukte als vermindernder Falktor hinzu. Infolge des ersten Zusatzes von Natriumnitrat zur Chlornatriumlösung wird die Menge des gelösten Chlornatriums demnach herabgedrückt, sowohl

1) Diese Zeitschr. 4, 372-383 (1889).

2) Diese Zeitschr. 6, 241-267 (1890); vgl. Noyes und Le BIanc a. a. O. $6,385-402(1890)$. 
durch die Verdünnung des Lösungsmittels, als auch - nach der Annahme von Nernst - durch die Zufuhr einer gewissen Menge freier Natriumatiome, die durch Dissociation des Natriumnitrats bei der Auflösung in Freiheit gesetzt sind. Ein weiterer Zusatz wird die Menge der aus Natriumnitrat abgespaltenen Ionen nicht wesentlich rermehren, weil ja der Dissociationsgrad der Lösungen von Elektrolyten mit zunehmender Konzentration erheblich abnimmt. Deshalb wirkt der erste Zusatz von Natriumnitrat stärker als ein gleichwertiger von Alkohol, weil bei letzterem wesentlich nur die verdïnnende Wirkung in Frage kommt, bei ersterem neben dieser noch die aus der Dissociationshypothese abgeleitete. Die ferneren Zusätze von Natriumnitrat erniedrigen die Löslichkeit nur durch ihre verdünnende Wirkung ebenso wie der Alkohol, und deshalb läuft die Kurve der Löslichkeitsverminderung nach dem ersten Zusat\% von Natriumnitrat nahezu parallel der bei Alkoholzusatz beobachteten. In welchem Masse die beiden Falktoren an der Löslichkeitsverminderung beteiligt sind, kann vielleicht experimentell ermittelt werden, wenn man die Beeinflussung der Löslichkeit von Kochsalz in Flüssigkeiten geringeren Lösungsvermögens, am besten in Weingeist wechselnder Konzentration, durch Zusätze von Natriumnitrat untersucht.

Abgesehen also von dem Einfluss der Dissociation wird bei den vier untersuchten Salzen die Löslichkeit durch Zusatz von Alkohol in gleicher Weise vermindert wie durch den Zusatz von indifferenten Salzen. Beide Versuchsreihen bestätigen also einander, und dadurch wird es sehr wahrscheinlich gemacht, dass die zunächst als empirische Regel aufgestellte Beziehung der Wassex- und Salzmengen in gleichen Volumen der gesättigten Lösungen zu einander eine allgemein gültige ist. Man wird als genügend erwiesen annehmen dürfen, dass dieselbe Menge Wasser in Berührung mit zwei darin löslichen Stoffen für jeden von beiden als Lösungsmittel dient, so als wenn der andere Stoff nicht zugegen wäre, und dass die Menge, die es von dem einen aufnimmt, nur insofern durch die Gegenwart des anderen beeinträchtigt ist, als diesex vermöge seiner Raumerfüllung das Lösungsmittel in einem grösseren Raume verteilt, vorausgesetzt, dass die beiden Stoffe sich chemisch nicht beeinflussen, miteinander keine Molekülverbindung bilden, einander nicht isomorph sind und kein gemeinsames Ion in wässeriger Lösung abspalten; in letzterem Falle werden die durch die Dissociationsgesetze bedingten Einflïsse auf die Löslichkeit sich in mehr oder minder grossem Massstabe bemerlkbar machen.

Die Versuche über die Verminderung der Löslichkeit des Natrium- 
nitrats durch Kochsalzzusatz haben die durch ganz andere Untersuchungen wahrscheinlich gewordene Thatsache, dass das in festem Zustande bei Temperaturen iiber $0^{\circ}$ unbeständige Hydrat $\mathrm{NaCl}+2 \mathrm{H}_{2} \mathrm{O}$ in Lösungen von höherer Temperatur beständig ist, bestätigt und damit für die Existenz von. Hydraten in Lösungen iiberhaupt einen nenen Beweis geliefert. Wichtige Aufschlüsse über die Zustände der Lösungen, über die Existenz von Hydraten und Doppelsalzen in denselben und über das Verhältnis isomorpher Substanzen zu einander sind ron weiteren Untersuchungen nach den hier angewandten Methoden zu erhoffen.

Clausthal, Bergakademie. 